\title{
Effects of atorvastatin and rosuvastatin on blood lipids, platelet aggregation rate and inflammatory factors in patients with cerebral infarction
}

\author{
Guo-jun Cao*, Xing-feng Zhang and Ke-da Zheng \\ Department of General Medicine, Feng Hua People's Hospital of Zhe Jiang Province, Zhe Jiang Feng Hua, 315500, China \\ *For correspondence: Email: gj0693@163.com \\ Sent for review: 18 August 2017 \\ Revised accepted: 28 September 2017
}

\begin{abstract}
Purpose: To investigate the effects of atorvastatin and rosuvastatin on blood lipids, platelet aggregation rate (PAR) and inflammatory factors in patients with cerebral infarction.

Methods: Patients $(n=120)$ with cerebral infarction treated in Feng Hua People's Hospital, Jiang Feng Hua, China from January 2014 to October 2016 were randomly divided into control group (clopidogrel combined with atorvastatin, 60 cases) and observation group (clopidogrel combined with rosuvastatin, 60 cases). Blood lipids, PAR, inflammatory factors and carotid atherosclerotic plaque were recorded and compared.

Results: Following treatment, total cholesterol (TC), triglycerides (TG) and low density lipoprotein cholesterol (LDL-C) in the observation group were significantly lower $(p<0.05)$ than in the control group, while high density lipoprotein cholesterol $(H D L-C)$ was significantly higher $(p<0.05)$. C-reactive protein (CRP), tumor necrosis factor- $\alpha$ (IL-6) and interleukin-6 (IL-6) were significantly decreased in the two groups after treatment $(p<0.05)$. Plaque area, intima-media thickness (IMT) and number of plaques in the two groups were significantly lower after treatment than before treatment $(p<0.05)$. Plaque area, IMT and number of plaques in the observation group were significantly lower than those in the control group $(p<0.05)$.

Conclusion: Atorvastatin and rosuvastatin have no significant effect on the antiplatelet function of clopidogrel, but rosuvastatin shows better control of blood lipids, carotid atherosclerosis and inflammatory factors.
\end{abstract}

Keywords: Atorvastatin, Rosuvastatin, Cerebral infarction, Blood lipids, Platelet aggregation rate, Inflammatory factors

Tropical Journal of Pharmaceutical Research is indexed by Science Citation Index (SciSearch), Scopus, International Pharmaceutical Abstract, Chemical Abstracts, Embase, Index Copernicus, EBSCO, African Index Medicus, JournalSeek, Journal Citation Reports/Science Edition, Directory of Open Access Journals (DOAJ), African Journal Online, Bioline International, Open-J-Gate and Pharmacy Abstracts

\section{INTRODUCTION}

Cerebral infarction is one of the common cerebrovascular diseases. With rising aging population in China, improvement in living standards and changes in work pressure, morbidity and mortality of stroke are on the increase [1]. Acute ischemic stroke refers to a cerebrovascular disease or recurrence of pathological blood disorder based on localized or diffuse brain dysfunction, and is the most common type of cerebral infarction (accounts for about $50 \sim 80 \%$ ). Its main clinical manifestations are hemiplegia, aphasia, and nerve function deficiency syndrome consciousness disorder [2,3].

In the acute stage of ischemic stroke, the main treatment measures are aimed at thrombolysis to restore blood circulation, protect nerve function 
and save the life of patient. There are many reports about the treatment measures in the acute phase; there are no controversies about these treatments. Related studies show that after the onset of cerebral infarction, the patient has a higher recurrence rate, up to $13.4 \%$ for 1-year recurrence rate [4], $26 \%$ for 5-year recurrence rate and $39 \%$ for 10-year recurrence rate [5]. Thus, high recurrence rate is a serious problem in current treatment of cerebral infarction. Treatment measures for ischemic stroke in the acute stage include antiplatelet therapy, regulation of blood lipids and blood pressure; and treatment of primary diseases [6]. Statins as lipid-lowering drugs are widely used in the later treatment of ischemic stroke. Studies have shown that statins not only play a role in regulating lipids, they also reduce inflammatory effects, reduce atherosclerotic plaque, and exert anti-platelet aggregation effect, all of which improve the prognosis of cerebral infarction $[7,8]$.

The commonly used drugs in late post-ischemic stroke include statins and clopidogrel. Atorvastatin is metabolized by cytochrome P450 (CYP3A4) and causes competitive inhibition leading to clopidogrel resistance, decreased antiplatelet activity and increased risk of cardiovascular and cerebrovascular events [9]. Rosuvastatin is metabolized by cytochrome P450 2C9 (CYP2C9), and has no significant effect on anti-platelet activity of clopidogrel. However, some studies reported that statins have no effect on the origin of clopidogrel resistance, and the impact of its anti-platelet effect remains controversial [9-11].

The research aimed to investigate the effects of atorvastatin and rosuvastatin on blood lipids, platelet aggregation rate (PAR) and inflammatory factors in patients with cerebral infarction.

\section{METHODS}

\section{Study subjects}

Patients with cerebral infarction (120) treated in Feng Hua People's Hospital of Zhe Jiang Province, Zhe Jiang Feng Hua, from January 2014 to October 2016, were selected. Inclusion criteria were: (1) Patients with clinical symptoms in line with "Clinical diagnostic criteria for stroke" by WHO [12]; (2) patients confirmed for presence of infarction by head CT or MRI examination; (3) patients aged 30 to 79 years with complete clinical data; (4) patients who did not take antiplatelet drugs or statins 3 months before the study; and (5) patients and their families who gave informed consent and signed consent form. The exclusion criteria were:
(1) patients with severe heart, kidney, liver and other vital organ dysfunction; (2) Patients with mental illness (3) Patients with contra-indications to clopidogrel, rosuvastatin and atorvastatin as well as patients allergic to pharmaceutical ingredients, pregnant patients and lactating patients. This study was approved by the ethics committee of Feng Hua People's Hospital (no. ZFH2013032), and followed the guidelines of Helsinki Declaration [13]. The recruited patients were divided into the control group and the observation group by random number table method, with 60 cases in each. In the control group, 35 cases were male and 25 were female. They ranged in age from 32 to 79 years (mean age, $45.83 \pm 7.49$ years). There were 28 cases with hypertension, 15 cases of hyperlipidemia, 10 cases of diabetes mellitus and 7 cases with smoking history. In the observation group, 30 cases were male and 30 were female. They were aged 33 to 77 years (mean age, $46.75 \pm 7.51$ years). There were 25 cases of hypertension, 16 cases of hyperlipidemia, 11 cases of diabetes mellitus and 8 cases wih smoking history. The differences in sex, age and basic disease between the two groups had no statistical significance $(p>0.05)$.

\section{Treatment protocol}

The two groups of patients received conventional treatment in accordance with recent "Chinese ischemic stroke and transient ischemic attack secondary prevention guidelines" [14]. This included blood glucose and blood pressure control, antiplatelet therapy and primary disease treatment. In the control group, patients were given clopidogrel combined with atorvastatin [clopidogrel (Sanofi Winthrop Industries) at a dose of $75 \mathrm{mg}$ orally once daily; and $20 \mathrm{mg}$ atorvastatin (Pfizer Ireland Pharmaceuticals) orally once a day. The patients in the observation group were given clopidogrel combined with rosuvastatin [clopidogrel (drug as in control group) $75 \mathrm{mg}$ once daily, orally; rosuvastatin (strength, $10 \mathrm{mg} /$ tablet, approval number: J20120006; Astra Zeneca UK limited) 10 mg, once a day, orally. The two groups of patients were treated continuously for 6 months.

\section{Observation parameters}

Levels of serum lipids, inflammatory factors and carotid atherosclerosis were measured 6 months before and after treatment. Fasting venous blood was taken early in the morning 1, 2, 3 and 6 months before and after treatment for PAR test. Total cholesterol (TC), triglycerides (TG), low density lipoprotein cholesterol (LDL-C) and high density lipoprotein cholesterol (HDL-C) levels of 
patients were measured by TBA-40FR fully automatic biochemical analyzer (Toshiba, Japan). Platelet aggregation rates were measured before treatment and after 1, 2, 3, and 6 months of treatment using Sonoclot coagulation and platelet function analyzers (Sienco Inc, USA). Immuno-turbidimetry was used for determination of C-reactive protein (CRP) levels. Interleukin -6 (IL-6) and tumor necrosis factor $-\alpha$ (TNF- $\alpha$ ) were determined on double antibody enzyme linked immunosorbent assay (ELISA) kits (Shanghai Senxiong Technology Industrial Co., Ltd).

The area and number of carotid plaque and carotid intima-media thickness (IMT) were determined on Color Doppler ultrasound (PHILPS, Holland). Bilateral common carotid artery (CCA), internal carotid artery (ICA) and plaques in the bifurcation of the CCA were recorded. Qualitative and quantitative records of ultrasound echo characteristics and plaque morphology were kept. Blood levels of LDH, CK$\mathrm{MB}, \mathrm{ALT}, \mathrm{AST}$, and total bilirubin were assayed in the two groups.

\section{Statistical analysis}

Statistical analysis is analyzed by SPSS version 20.0 and the data are presented as mean \pm standard deviation (SD) and, were compared between the two groups using t-test. Enumeration data are presented as frequency or percentage $(\%)$, and were compared using $X^{2}$ test. $P<0.05$ was considered statistically significant.

\section{RESULTS}

\section{Blood lipid levels}

There were no statistically significance in $\mathrm{TC}$, TG, HDL-C and LDL-C levels between the two groups before the treatments $(p>0.05)$. However, after the treatments, TC, TG and LDL$\mathrm{C}$ levels were significantly decreased $(p<0.05)$, while HDL-C levels increased significantly $(p<$ 0.05 ) in the two groups. The levels of TC, TG and LDL-C in the observation group after treatment were significantly lower than corresponding values in the control group $(p<$ $0.05)$. HDL-C was significantly higher than in the control group $(p<0.05$; Table 1$)$.

\section{Platelet aggregation rate}

There was no significant difference in PAR between the two groups before treatment $(p>$ $0.05)$, but PAR in the two groups decreased significantly $(p<0.05)$ after treatment. However, PAR values (in the two groups) after treatment for 1, 2, 3 and 6 months were similar $(p>0.05$; Table 2).

\section{Levels of inflammatory factors}

As shown in Table 3, differences in the levels of inflammatory factors between the two groups before treatment was not statistically significant $(p>0.05)$.

Table 1: Blood lipid levels (mmol/L) of the patients

\begin{tabular}{|c|c|c|c|c|c|}
\hline Group & Time & TC & TG & HDL-C & LDL-C \\
\hline \multirow{4}{*}{ Control group (60) } & Before treatment & $5.88 \pm 1.09$ & $2.07 \pm 0.37$ & $0.87 \pm 0.22$ & $3.75 \pm 0.77$ \\
\hline & After treatment & $5.11 \pm 1.01$ & $1.67 \pm 0.21$ & $1.11 \pm 0.34$ & $2.91 \pm 0.61$ \\
\hline & $T$ & 4.014 & 7.283 & 4.591 & 6.624 \\
\hline & $P$ & 0.000 & 0.000 & 0.003 & 0.011 \\
\hline \multirow{4}{*}{$\begin{array}{l}\text { Observation group } \\
(60)\end{array}$} & Before treatment & $5.97 \pm 1.12$ & $2.11 \pm 0.42$ & $0.84 \pm 0.21$ & $3.88 \pm 0.79$ \\
\hline & After treatment & $4.52 \pm 0.73^{\pi}$ & $1.34 \pm 0.19^{\pi}$ & $1.35 \pm 0.42^{\pi}$ & $2.31 \pm 0.47^{\star}$ \\
\hline & $T$ & 7.011 & 1.989 & 8.413 & 13.230 \\
\hline & $P$ & 0.000 & 0.000 & 0.000 & 0.000 \\
\hline
\end{tabular}

Values are expressed as mean $\pm S D ; p<0.05$, compared to control group after treatment

Table 2: Platelet aggregation rate (\%) of the patients

\begin{tabular}{lccccc}
\hline Group & $\begin{array}{c}\text { Before } \\
\text { treatment }\end{array}$ & $\begin{array}{c}\text { 1-month } \\
\text { treatment }\end{array}$ & $\begin{array}{c}\text { 2-month } \\
\text { treatment }\end{array}$ & $\begin{array}{c}\text { 3-month } \\
\text { treatment }\end{array}$ & $\begin{array}{c}\text { 6-month } \\
\text { treatment }\end{array}$ \\
\hline Control group (60) & $70.32 \pm 14.01$ & $50.87 \pm 13.95$ & $48.11 \pm 12.43$ & $43.54 \pm 13.07$ & $41.47 \pm 13.04$ \\
Observation group & $71.17 \pm 14.88$ & $51.34 \pm 13.07^{*}$ & $47.35 \pm 12.12^{*}$ & $41.18 \pm 12.84^{\star}$ & $39.71 \pm 12.94^{\star}$ \\
$(60)$ & 0.322 & 0.190 & 0.339 & 0.998 & 0.742 \\
$\mathrm{~T}$ & 0.748 & 0.849 & 0.735 & 0.320 & 0.460 \\
$P$ & & 0.050 & &
\end{tabular}

Values are expressed as mean $\pm S D ; p<0.05$ compared with values before treatment 
Table 3: Levels of inflammatory factors in the patients

\begin{tabular}{llccc}
\hline Group & Time & CRP $(\mathbf{m g} / \mathbf{L})$ & TNF- $\mathbf{\alpha}(\mathbf{p g} / \mathbf{m L})$ & IL-6 $(\mathbf{p g} / \mathbf{m L})$ \\
\hline \multirow{4}{*}{ Control (60) } & Before treatment & $14.87 \pm 2.97$ & $185.74 \pm 27.41$ & $139.88 \pm 16.89$ \\
& After treatment & $8.68 \pm 1.45$ & $90.14 \pm 25.17$ & $108.74 \pm 13.71$ \\
& $T$ & 14.507 & 19.899 & 11.088 \\
& $P$ & 0.000 & 0.000 & 0.000 \\
Observation (60) & Before treatment & $15.11 \pm 3.07$ & $190.17 \pm 26.33$ & $140.58 \pm 17.47$ \\
& After treatment & $5.79 \pm 1.31^{*}$ & $80.04 \pm 19.25^{*}$ & $100.97 \pm 9.87^{*}$ \\
& $T$ & 21.629 & 26.154 & 15.291 \\
& $P$ & 0.000 & 0.000 & 0.000 \\
\hline
\end{tabular}

Values are expressed as mean $\pm S D ; p<0.05$, compared with control group after treatment

Table 4: Carotid atherosclerotic plaques in the patients

\begin{tabular}{llccc}
\hline Group & Time & $\begin{array}{c}\text { Plaque area } \\
\left(\mathbf{m m}^{2}\right)\end{array}$ & IMT(mm) & $\begin{array}{c}\text { Number of } \\
\text { plaques (个) }\end{array}$ \\
\hline Control $(\mathrm{n}=60)$ & Before treatment & $15.74 \pm 2.14$ & $1.74 \pm 0.67$ & $2.63 \pm 1.07$ \\
& After treatment & $11.14 \pm 1.87$ & $1.34 \pm 0.51$ & $1.74 \pm 0.84$ \\
& $T$ & 12.538 & 3.680 & 3.665 \\
& $P$ & 0.000 & 0.008 & 0.008 \\
Observation $(\mathrm{n}=$ & Before treatment & $16.41 \pm 2.42$ & $1.68 \pm 0.61$ & $2.35 \pm 1.14$ \\
$60)$ & After treatment & $10.37 \pm 1.74$ & $1.01 \pm 0.37$ & $1.42 \pm 0.24$ \\
& $T$ & 1.982 & 7.274 & 8.547 \\
& $P$ & 0.000 & 0.000 & 0.000 \\
\hline
\end{tabular}

Values are expressed as mean $\pm S D ; p<0.05$, compared with control group after treatment

After treatment, CRP, TNF- and IL-6 levels in the two groups were significantly lower than the corresponding values before treatment $(p<$ 0.05), while the levels of CRP, TNF- and IL-6 in the observation group were significantly lower than those in the control group $(p<0.05)$.

\section{Carotid atherosclerotic plaques}

Plaque area, IMT and number of plaques were similar in the two groups before treatment. However, after treatment, the plaque area, IMT and number of plaques decreased significantly in the two groups $(p<0.05)$, while in the observation group, plaque area, IMT and number of plaques were significantly lower than those in the control group $(p<0.05$; Table 4$)$.

\section{Recurrence rate and complications}

After 6 months of treatment in the control group, one case of recurrent cerebral infarction $(1.67 \%$; $1 / 60)$; one case of liver injury $(1.67 \% ; 1 / 60)$ and 2 cases of increased myocardial enzymes (3.33 $\% ; 2 / 60$ ) were seen, along with 1.67 and $5 \%$ recurrence rate and complication rate, respectively. There was no recurrence in the observation group, but 2 cases of liver injury (3.33 \%; 2/60) and 3 cases of elevated myocardial enzymes (5\%; 3/60) occurred, while the total incidents of complications was $8.33 \%$ $(5 / 60)$. Adverse reactions of the two groups got better after their respective treatments, and no other serious adverse reactions were observed. Differences in recurrence rate and total incidence of complications between the two groups did not show statistical significance $(p>0.05)$.

\section{DISCUSSION}

Cerebral infarction is a common disease in neurology, with acute onset and speedy development. In recent years, the incidence has increased in younger populations, seriously affecting the health and quality of life of patients [15]. Atherosclerosis is the pathological basis of cerebral infarction, and the main risk factor of atherosclerotic plaque formation is high blood lipids. Thus, lipid-lowering therapy can effectively reduce atherosclerosis and the incidence of cerebral infarction [16]. Clopidogrel combined with statins has become a routine treatment for prevention of cerebral infarction. It can effectively reduce the risk of recurrence and improve prognosis in patients with cerebral infarction [17]. Clopidogrel, one of the most common antiplatelet agents, is metabolized by the CYP450 enzyme system to form an active component, which can irreversibly bind to the ADP receptor on the platelet membrane surface, thereby exerting its antiplatelet aggregation effect[18].

CRP, TNF- alpha and IL-6 belong to non-specific markers of the acute stage of systemic inflammatory response, which increase blood viscosity, promote plaque formation and accelerate atherosclerosis [19]. They are risk factors for atherosclerosis. CRP is an acute phase protein synthesized in the liver. It is involved in the inflammatory process of 
atherosclerosis, by inducing increases in intimal thickness and rupture of plaques, which result in acute cerebral infarction. Statins are hydroxymethylglutaryl coenzyme $A$ reductase (HMG-CoA) inhibitors which can block cholesterol biosynthesis and lipid peroxidation, stabilize atherosclerotic plaque and increase cerebral blood flow [20,21].

It has been reported that $30 \%$ of ischemic stroke are caused by carotid artery diseases, and that cerebral infarction is closely related to carotid atherosclerosis [22]. Atherosclerosis involves the intracranial and external arteries. Lipids gradually accumulate in the arterial smooth muscle cells in the vascular smooth muscle, leading to intimal hyperplasia and plaque formation. Inflammation and oxidative stress lead to plaque rupture and arterial invasion, blockage of the lumen by thrombosis and ischemic cerebral infarction $[23,24]$. Thus, there is a complementary relationship between the level of inflammation and carotid atherosclerotic plaque formation. This implies that plaque formation can be controlled by reducing the level of inflammation, thereby preventing cerebral infarction.

In the current study, the level of inflammatory factors and carotid atherosclerosis were significantly decreased in the observation group, when compared with their corresponding values before treatment. This finding is due to the fact that statins can lower the level of CRP, inhibit platelet aggregation, promote fibrinolysis and stabilize atherosclerotic plaque. Besides, rosuvastatin suppresses aggregation of inflammatory cells and the formation of foam cells and oxygen free radical produced by macrophages $[25,26]$.

Comparison of the two kinds of statin in patients with cerebral infarction revealed that platelet aggregation rate and blood lipid levels were lower than those before treatment, suggesting that the two statins had lipid-lowering and antiplatelet effects. However, the data showed that blood lipid levels in the observation group changed greatly when compared with the control group, while in the two groups, platelet aggregation rate declined to the same extent. This shows that rosuvastatin was more effective than atorvastatin in reducing blood lipids in cerebral infarction. The mechanism of action is based on the lipid-lowering effect of statins and their inhibitory effects on the key enzymes of cholesterol biosynthesis, thereby regulating the rate of synthesis of cholesterol and inhibiting atherosclerosis [27].
Rosuvastatin has a stronger inhibitory effect on HMG-CoA reductase than any other statin; it has a long half-life, low hepatic first pass effect, and better pharmacokinetic properties [28]. Studies by Johansen et al [29] and Wang et al [30] demonstrated that agents metabolized by CYP3A4 (such as atorvastatin) are lipophilic, and so promote clopidogrel resistance, while rosuvastatin which is not metabolized by CYP3A4, is hydrophilic, and has obvious influence on the platelet aggregation effect of clopidogrel. This study found that the platelet aggregation functions of clopidogrel in the patients with secondary prevention of cerebral infarction in the two groups may be similar, possibly due to multiple metabolic pathways of clopidogrel.

\section{Study limitations}

There are some deficiencies in this study. All subjects were in-patients admitted to the hospital, and the sample size was small. Thus, there may be selective bias. Future studies should use an expanded sample size, stratify the patients, and extend follow-up to obtain more reliable conclusions.

\section{CONCLUSION}

The results obtained in this study indicate that atorvastatin and rosuvastatin have good lipidlowering and anti-inflammatory effects in patients with cerebral infarction, without significant effects on the platelet aggregation function of clopidogrel. The results also revealed that rosuvastatin displays better control of blood lipids, atherosclerosis and levels of inflammatory factors, and thus possesses distinct therapeutic benefits.

\section{DECLARATIONS}

\section{Acknowledgement}

None.

\section{Conflict of Interest}

No conflict of interest associated with this work.

\section{Contribution of Authors}

The authors declare that this work was done by the authors named in this article and all liabilities pertaining to claims relating to the content of this article will be borne by them. 


\section{Open Access}

This is an Open Access article that uses a funding model which does not charge readers or their institutions for access and distributed under the terms of the Creative Commons Attribution License (http://creativecommons.org/licenses/by/ 4.0) and the Budapest Open Access Initiative (http://www.budapestopenaccessinitiative.org/rea d), which permit unrestricted use, distribution, and reproduction in any medium, provided the original work is properly credited.

\section{REFERENCES}

1. Song $X$, Xue S, Zhao J, Wu J. Screening for Fabry's disease in young patients with ischemic stroke in a Chinese population. Int J Neurosci 2017; 127(4): 350355.

2. Xing $Y$. Guidelines for the diagnosis and treatment of acute ischemic stroke in China 2010. Chin Clin Dr 2011; 43(3): 67-73.

3. Flint AC, Kamel H, Navi BB, Rao VA, Faigeles BS, Conell $C$, Klingman JG, Sidney S, Hills $N K$, Sorel $M$, et al. Statin use during ischemic stroke hospitalization is strongly associated with improved poststroke survival. Stroke 2012; 43(1): 147-154.

4. Chen YX, Xiang CX, Xie RX, Zhang JH, Song CY, Cheng $N$, Wang SJ, Sun YH, Han SL. The predictive value of Essen stroke risk scale in the recurrence of ischemic stroke patients. Chin J Mod Med 2014; 24(17): 99-102.

5. Liu J, Yun Z, Wu Y, Yan L, Teng Z, Hao Y. Association of carotid atherosclerosis and recurrent cerebral infarction in the Chinese population: a meta-analysis. Neuropsychiatr Dis Treat 2017; 13(1): 527-533.

6. Mouthonreignier $C$, Bonnaud $I$, Gaudron $M$, Vannierbernard S, Bodin JF, Cottier JP, De TB, Debiais S. Impact of a direct-admission stroke pathway on delays of admission, care, and rates of intravenous thrombolysis. Rev Neurol 2016; 172(12): 756-760.

7. Bener A, Dogan M, Barakat L, Alhamaq AO. Comparison of efficacy, safety, and cost-effectiveness of various statins in dyslipidemic diabetic patients. Indian $J$ Pharmacol 2014; 46(1): 88-93.

8. Sun $L F, A n D Q$. Progress in the pleiotropic effects of statins on anti-atherosclerotic mechanisms. Chin $J$ Integr Med Cardio 2015; 13(4): 453-457.

9. Geng YP, Xie RQ, Cui W, Liu F, Yang XC, Zhang JD, Yang $X H, G u G Q$, Yang J. The influence of atorvastatin or rosuvastatin on antiplatelet activity of clopidogrel. Chin J Geriatr Heart Brain Ves Dis 2011; 13(1): 27-29.

10. Xu HY, Liu D, Wang WG, Kong LT. Research progress of interaction between statins and common cardiovascular drugs. Chinese pharmacy 2016; 11(1): 1582-1584.

11. Yang MY, Han B, Tian $L Y, X u Y, X U$ J, Qiang YW. Effects of atorvastatin and rosuvastatin on antiplatelet activity of clopidogrel in elderly patients with acute coronary syndrome. Acad J Chin PLA Med Sch 2015; 36(12): 1208-1210.

12. Chinese Society of Neurology. Guidelines for the diagnosis and treatment of acute ischemic stroke in China 2014. Chin J Neurol 2015; 48(4): 246-257.

13. Czarkowski M. Helsinki Declaration--next version. Pol Merkur Lekarski 2014; 36(215): 295-297.

14. Chinese Society of Neurology, Cerebral vascular group. Secondary guidelines for prevention of ischemic stroke and transient ischemic attacks in China (2010). Chin J Neurol 2015; 48(4): 154-160.

15. Van MT, Nederkoorn PJ, Hb VDW, Stam J, Richard E. Quality of life after surgical decompression for spaceoccupying middle cerebral artery infarction: Systematic review. Int J Stroke 2015; 10(2): 170-176.

16. Xiao ZC, Chen HH, Gang LI. Research on stability of carotid atherosclerotic plaques and Vascular endothelial function in patients with acute cerebral infarction. J Apop/ Nerv Dis 2013; 30(5): 422-424.

17. Lu M. Report: Impact of drug combination of clopidogrel and pantoprazole In the prognosis of patients with transient ischemic attack. Pak J Pharm Sci 2017; 30(1): 217-221.

18. Pan $Y$, Chen $W, X u Y$, Yi X, Han $Y$, Yang $Q$, Li X, Huang LA, Johnston SC, Zhao X, et al. Genetic Polymorphisms and Clopidogrel Efficacy for Acute Ischemic Stroke or Transient Ischemic Attack : A Systematic Review and Meta-Analysis. Circulation 2017; 135(1): 21-33.

19. Eggers AE. A suggestion about the cause of inflammation in acute atherosis complicating poor placentation in preeclampsia. Med Hypotheses 2015; 85(6): 718-719.

20. Raghow R. Statins redux: A re-assessment of how statins lower plasma cholesterol. World J Diabetes 2017; 8(6): 230-234.

21. Li XS, Jia XW, Wang YF, Gao SX. New perspectives on statins pleiotropic effects. Chin J Card Review 2016; 14(4): 309-313.

22. Guo $X Z$, $X u$ QS, Lu ZH, Liu HX, Li JH, Feng JY, Sun $X X$, Zhao XC, Zhang JF. Clinical study of carotid plaques treated with ultrasound. Chi J Emerg Resusc and Disaster Med 2012; 7(9): 885-886.

23. Nakano R, Takebe N, Ono M, Hangai M, Nakagawa R, Yashiro S, Murai T, Nagasawa K, Takahashi Y, Satoh J, et al. Involvement of oxidative stress in atherosclerosis development in subjects with sarcopenic obesity. Obes Sci Pract 2017; 3(2): 212-218.

24. Wang J. Diagnosis and treatment strategy of carotid atherosclerosis in the elderly. Chin J Geriatr Heart Brain Vessel Dis 2015; 17(1): 109-110.

25. Kwon O, Kang SJ, Kang SH, Lee PH, Yun SC, Ahn JM, Park DW, Lee SW, Kim YH, Lee CW, et al. Relationship Between Serum Inflammatory Marker Levels and the Dynamic Changes in Coronary Plaque Characteristics After Statin Therapy. Circ Cardiovasc Imaging 2017; 10(7): e005934. 
26. Wu WB. Effect of rosuvastatin on levels of plasma inflammatory cytokines in patients with acute cerebral infarction. J Clin Med Pract 2015; 19(9): 134-136.

27. Neary NM, Booker OJ, Abel BS, Matta JR, Muldoon N, Sinaii N, Pettigrew RI, Nieman LK, Gharib AM. Hypercortisolism is associated with increased coronary arterial atherosclerosis: analysis of noninvasive coronary angiography using multidetector computerized tomography. J Clin Endocrinol Metab 2013; 98(5): 20452052.
28. Zhang LL. Clinical study of rosuvastatin and simvastatin in elderly patients with lower extremity atherosclerosis. Jilin University 2014.

29. Johansen ME, Hefner JL, Foraker RE. Antiplatelet and Statin Use in US Patients With Coronary Artery Disease Categorized by Race/Ethnicity and Gender, 2003 to 2012. Am J Cardiol 2015; 115(11): 1507-1512.

30. Wang YC, Hsieh TC, Chou CL, Wu JL, Fang TC. Risks of Adverse Events Following Coprescription of Statins and Calcium Channel Blockers: A Nationwide PopulationBased Study. Medicine 2016; 95(2): e2487. 